INPLASY

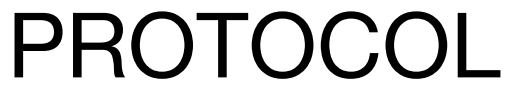

To cite: Li et al. Efficacy and Safety of Trilaciclib in Patients With Bone marrow suppression caused by chemotherapy: A Systematic Review and MetaAnalysis of Randomized Controlled Trials. Inplasy protocol 202150021. doi: 10.37766/inplasy2021.5.0021

Received: 05 May 2021

Published: 05 May 2021

Corresponding author: Li Xiaoyun

Ixy092979@163.com

Author Affiliation:

Southwest Medical University

Support: Nos. 81774013 and 8180422.

Review Stage at time of this submission: Preliminary searches.

Conflicts of interest:

None declared.

\section{Efficacy and Safety of Trilaciclib in Patients With Bone marrow suppression caused by chemotherapy: A Systematic Review and MetaAnalysis of Randomized Controlled Trials}

$$
\text { Li, XY1; Li, H²; Li, ZP3; Wu, JM; Jiang, ZM5. }
$$

Review question / Objective: P: Patients with bone marrow suppression; I: Treat with trilaciclib ; C: Treat without trilaciclib ; O: duration of severe neutropenia ( $\mathrm{SN}$; defined as absolute neutro-phil count <0.5 $\times$ 109cells per L) in Cycle 1 and occurrence of SN during the treatment period; S: RCT s.

Condition being studied: Trilaciclib is an intravenous CDK4/6 inhibitor administered prior to chemotherapy to preserve haematopoietic stem and progenitor cells and immune system function from chemotherapy-induced damage (myelopreservation). But at present, there is no systematic evaluation report on its therapeutic effectiveness and safety. This protocol aims to reveal the efficacy and safety of trilaciclib for preserving haematopoietic stem and progenitor cells and immune system function from chemotherapy-induced damage.

INPLASY registration number: This protocol was registered with the International Platform of Registered Systematic Review and Meta-Analysis Protocols (INPLASY) on 05 May 2021 and was last updated on 05 May 2021 (registration number INPLASY202150021).

\section{INTRODUCTION}

Review question / Objective: P: Patients with bone marrow suppression; I: Treat with trilaciclib ; C: Treat without trilaciclib ; $O$ : duration of severe neutropenia (SN; defined as absolute neutro-phil count $<0.5$ $\times$ 109cells per $L$ ) in Cycle 1 and occurrence of SN during the treatment period; S: RCTs.
Condition being studied: Trilaciclib is an intravenous CDK4/6 inhibitor administered prior to chemotherapy to preserve haematopoietic stem and progenitor cells and immune system function from chemotherapy-induced damage (myelopreservation). But at present, there is no systematic evaluation report on its 
therapeutic effectiveness and safety. This protocol aims to reveal the efficacy and safety of trilaciclib for preserving haematopoietic stem and progenitor cells and immune system function from chemotherapy-induced damage.

\section{METHODS}

Participant or population: The inclusion criteria for the study will include: (1) studies with cancer patients who use chemotherapy as a treatment; (2) conference abstracts were only included when they provided adequate relevant information for assessment; (3) the patients undergoing chemotherapy were divided into two groups (treated with trilaciclib or without trilaciclib); Exclusion criteria will include: patients with incomplete data.

Intervention: Treat with trilaciclib.

Comparator: Treat without trilaciclib.

Study designs to be included: RCTs.

Eligibility criteria: The inclusion criteria for the study will include: (1) studies with cancer patients who use chemotherapy as a treatment; (2) conference abstracts were only included when they provided adequate relevant information for assessment; (3) the patients undergoing chemotherapy were divided into two groups (treated with trilaciclib or without trilaciclib); Exclusion criteria will include: patients with incomplete data.

Information sources: We will search the Medline, Embase, PubMed, ClinicalTrials.gov and Cochrane Library from inception to October 31, 2021 to retrieve relevant studies. We will also search citations of relevant primary and review. Authors of abstract in the meeting will be further searched in PubMed for potential full articles. $T$ o minimize the risk of publication bias, we will conduct a comprehensive search that included strategies to find published and unpublished studies.
Main outcome(s): Duration of severe neutropenia ( $\mathrm{SN}$; defined as absolute neutro-phil count $<0.5 \times 109$ cells per $L$ ) in Cycle 1 and occurrence of SN during the treatment period.

Quality assessment / Risk of bias analysis: Risk of bias assessment will be carried out according to the Newcastle-Ottawa Scale (NOS) to rate the internal validity of the individual studies, and funnel plots will be constructed to assess the risk of publication bias.

Strategy of data synthesis: All pairwise meta - analytic calculations will be performed with Review Manager software ( Rev Man ) version 5.3 (Cochrane Collaboration). Heterogeneity will be examined by computing the $Q$ statistic and $I^{2}$ statistic, and presence of reporting bias by visual inspection of funnel plots. Statistical significance was considered when the $P$ value $<0.05$.

Subgroup analysis: Patients undergoing chemotherapy treated with trilaciclib and patients undergoing chemotherapy treated without trilaciclib.

Sensitivity analysis: All pairwise meta analyticcalculations will be performed with Review Manager software ( Rev Man ) version 5.3 (Cochrane Collaboration). Heterogeneity will be examined by computing the $Q$ statistic and $I^{2}$ statistic, and presence of reporting bias by visual inspection of funnel plots. Statistical significance was considered when the $P$ value $<0.05$.

Country(ies) involved: China.

Keywords: Myelosuppression; Trilaciclib; chemotherapy.

Contributions of each author:

Author 1 - Li Xiaoyun.

Author 2 - Li Huan.

Author 3 - Li Ziping.

Author 4 - Wu Jianming.

Author 5 - Jiang Zhongmei. 